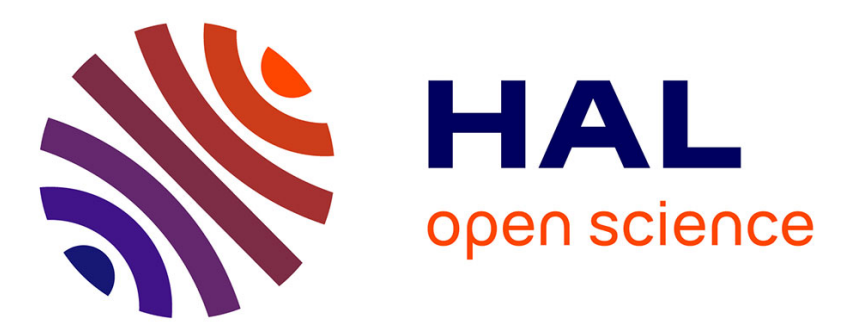

\title{
A purely model-based approach to object pose and size estimation with electric sense
}

Stéphane Bazeille, Vincent Lebastard, Frédéric Boyer

\section{To cite this version:}

Stéphane Bazeille, Vincent Lebastard, Frédéric Boyer. A purely model-based approach to object pose and size estimation with electric sense. IEEE Transactions on Robotics, 2020, pp.1-8. 10.1109/TRO.2020.3000285 . hal-02635296

\section{HAL Id: hal-02635296 \\ https://hal.science/hal-02635296}

Submitted on 27 May 2020

HAL is a multi-disciplinary open access archive for the deposit and dissemination of scientific research documents, whether they are published or not. The documents may come from teaching and research institutions in France or abroad, or from public or private research centers.
L'archive ouverte pluridisciplinaire HAL, est destinée au dépôt et à la diffusion de documents scientifiques de niveau recherche, publiés ou non, émanant des établissements d'enseignement et de recherche français ou étrangers, des laboratoires publics ou privés. 


\title{
A purely model-based approach to object pose and size estimation with electric sense
}

\author{
Stéphane Bazeille*, Vincent Lebastard ${ }^{\dagger}$, Frédéric Boyer ${ }^{\dagger}$ \\ * IRIMAS - Université de Haute-Alsace, 61 rue Albert Camus, Mulhouse, France. \\ ${ }^{\dagger}$ LS2N - IMT Atlantique, 4, rue Alfred Kastler, Nantes, France \\ Corresponding author email: stephane.bazeille@uha.fr
}

\begin{abstract}
In the 50s, biologists discovered that some electric fish are capable of discriminating the pose as well as the electric and geometric properties of surrounding objects by navigating and measuring the distortions of a self-generated electric field. In this paper, we address the challenging issue of ellipsoidal objects pose and size estimation for underwater robots equipped with artificial electric sense. Unlike current methods, the approach can estimate both the position and size in parallel with a single straight trajectory. No multi-polarization nor reactive self-alignment control are necessary to locate the object. The approach is a purely model-based heuristic that selects the best ellipsoid parameters among a set of potential candidates. It is based on a set of 4 electric measurements recorded at several positions along the robot trajectory along which the displacement is measured. The efficiency of the method is assessed over numerous experiments with different objects, several positions, and orientations, and two different kinds of water (fresh and salt water). Despite some model simplifications and experimental errors, location and size estimation errors are on average below $1 \mathrm{~cm}$ and $15 \%$ respectively, while offering promising perspectives for real-time computation.
\end{abstract}

Index Terms-Electric sense, object location, size estimation.

\section{INTRODUCTION}

$\mathbf{E}$ Lectric sense (or E-sense) is a sensorial ability used almost exclusively by some aquatic animals [1], [2]. Some fish can passively sense fluctuations of the nearby electric fields (passive E-sense), while others such as the elephant nose fish can sense the distortions of a self-generated electric field (active E-sense). Using E-sense these nocturnal fish can efficiently navigate in the confined turbid waters of equatorial forests. Behavioral experiments have shown that they can also perform complex cognitive tasks such as discriminating the size, electric properties and shape of objects [3], [4]. This ability is remarkable since the location and object properties are nonlinearly intricated in the electrical measurements [5]. In this paper, we address this difficult inverse problem with an underwater sensor equipped with E-sense.

\section{Previous WORK ON ELECTRIC SENSE}

In underwater robotics, artificial E-sense has been developed to supplement conventional sensors (sonar or vision) in harsh conditions. For instance, in turbid waters, the multiple echoes

This research was funded by European Union Horizon 2020 research and innovation program under grant agreement No 640967: EU-FET proactive project subCULTron (SUBmarine Cultures perform Long-Term Robotic Exploration of unconventional environmental Niches) http://www.subcultron.eu. due to suspended particles make the sonar signals difficult to interpret and the light absorption or scattering reduces drastically the performances of vision [6]. E-sense effectiveness has been proven for reactive navigation [7], object electrolocation [8], [9] or underwater docking [10]. According to our knowledge, in the field of E-sense based object recognition, only three contributions were proposed so far. Bai et al. [11] proposed an approach combining reactive navigation and a learning procedure to estimate the shape and the pose of an ellipsoidal object with an underwater sensor [12]. This sensor is an axisymmetric insulating shell with electrodes located at its extremities. Setting the emitters under voltage generates an electric field or "E-field". The perturbations of the E-field by the object are measured through the voltage between receiving electrodes. Exploiting the symmetries of the sensor and the object in the same equatorial plane, the approach first iterates reactive maneuvers to align the robot with the object. In a second phase, some maps built beforehand in simulation are used to determine the ellipsoid aspect ratio and the object's lateral distance and size using an equivalent sphere model and a Gaussian process regression. The second contribution was proposed by Lanneau et al. [13]. Similarly, the location and size estimation of ellipsoidal objects were separated and solved in cascade. Object location was obtained through reactive self-alignment or multiple signal classification method (MUSIC) [14], an algorithm that requires multi-polarization of the sensor to enrich the electric measurements recorded at different positions around the object. Once electrolocation is performed, the geometry and orientation of an object were estimated with a least-square estimation of its polarization tensor [15], coupled with an analytical model of the sensor measurements introduced in [7]. To close this state of the art, in [9], electrolocation and volume estimation of different objects were performed with an Unscented Kalman Filter and an analytical model of the electric response of spherical objects. All these methods gave good results. However, they require complex sensor motions.

In contrast, we here propose a purely model-based heuristic that estimates the pose and size of an ellipsoidal object with a single straight trajectory. Requiring no reactive control nor multi-polarization, the approach is relevant for semantic mapping purposes since objects can be detected, located and recognized as a background task while navigating for other purposes. This heuristic is based on a simple new score 


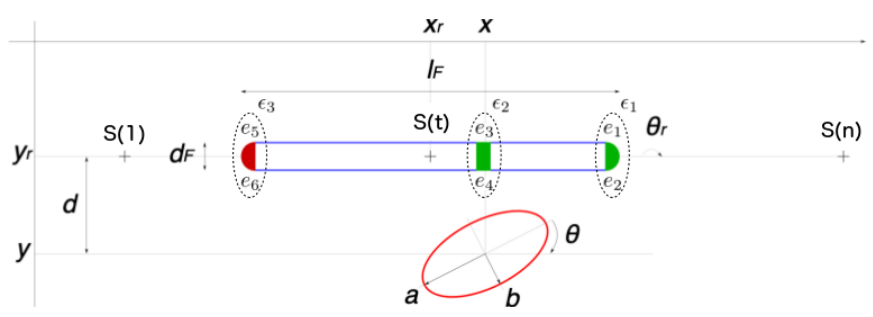

(a)

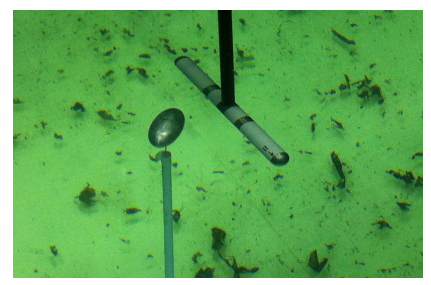

(b)

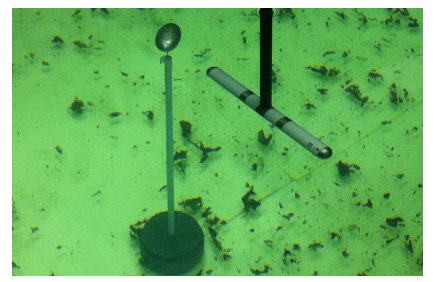

(c)
Fig. 1. Location and size estimation experiment. (a) The sensor performs a $60 \mathrm{~cm}$ straight trajectory from the pose $S(1)$ to $S(n)$. The robot poses are defined by: $S(t)=\left(x_{r}(t), y_{r}(t), \theta_{r}(t)\right)$. The ellipsoidal object is located at $30 \mathrm{~cm}$ from $\mathrm{S}(1)$ and at a lateral distance $d$ from the trajectory. It has a size: $a, b$, a pose: $x, y, \theta$ and an electrical property: $\gamma_{o}$. (b) The tank is a $4 \mathrm{~m}^{3}$ one, filled with salt water $(\gamma=1.6 \mathrm{~S} / \mathrm{m})$ and equipped with a gantry on which the sensor is attached. (c) The object is mounted on a small steel needle which is glued to a plastic rod laid on the tank floor.

function designed for quantifying the error between experimental measurements and those predicted by a model. The score is then used as a comparison criterion to select among a set of candidate parameters, those that best fit with the measurements. Beyond object estimation, this score can also be used to assess the consistency of the predictions of different models with a given database of measurements. A preliminary version of this idea was presented in [16]. Here, we refine the score function (see Sec. III), supplement the heuristic with an object detection phase (see Sec. IV), optimize the algorithm to decrease its computational time, and largely enriche the experimental section to clearly illustrate the efficiency of our method for objects of different geometry (spheres and ellipsoids), different poses, and with two water conductivities: a fresh water with conductivity $\gamma=0.04 \mathrm{~S} / \mathrm{m}$ and a salt water $(\gamma=1.6 \mathrm{~S} / \mathrm{m})$ with a concentration of salt about $10 \mathrm{~g} / \mathrm{L}$ (see Sec. V). While we obtained estimation results close to those of the existing methods, we are capable of estimating the size (aspect ratio and volume) of an ellipsoidal object without its prior location. Moreover, according to our knowledge, this is the second time after [8], that E-sense is used for underwater perception in salt water, and the first, for geometry estimation.

\section{CONTEXT OF THE STUDY}

\section{A. Description of the sensor and of its experimental use}

In all this article, we use a slender sensor presented in [17] (see Fig. 1). This sensor consists of a plastic cylinder equipped with 6 electrodes $e_{1}, \ldots, e_{6}$ grouped into pairs that belong to 3 macro-electrodes $\epsilon_{1}, \ldots, \epsilon_{3}$. Its length and diameter are $l_{F}=22 \mathrm{~cm}$ and $d_{F}=2 \mathrm{~cm}$ respectively. The 3 macroelectrodes are arranged along the sensor axis at $-11 \mathrm{~cm}, 3.5 \mathrm{~cm}$, and $11 \mathrm{~cm}$. This sensor was designed to provide an analytical model of measurements [18] (see Sec. III-B) while ensuring an E-field of dipolar shape mimicking that of the fish. The Efield around the sensor is generated by setting the tail macroelectrode (emitter) under voltage with respect to the other grounded electrodes (receivers). A voltage $U=2 \mathrm{~V}$ is set at a frequency $f_{r}=15 \mathrm{kHz}$ with an off-board sine wave generator. The electric currents that flow across the 4 receivers are measured at the frequency $f_{p}=66 \mathrm{~Hz}$ with an ampere meter circuit [17]. Note that only the amplitude of the electric currents is measured (the phase is not considered). To each recorded measurement, we assigned an index $t$ with $t \in \mathbb{N}$ and $t_{0}=1$. At the end of the trajectory: $t=n$. The 4 electric measurements on $e_{1}, \ldots, e_{4}$ are noted $I_{1}(t), \cdots, I_{4}(t)$. The sensor poses are recorded along the trajectory $S$ in a global reference frame, and noted $S(t)=\left(x_{r}(t), y_{r}(t), \theta_{r}(t)\right)$. For each macro-electrode $\epsilon$, the 2 currents are first filtered in the Fourier domain to remove the noise (cutoff frequency $2 \mathrm{kHz}$ ), before being combined to create more informative signals (see Fig. 2) noted $\delta I_{\mathrm{ax}}$ and $I_{\text {lat }}$. The currents $\delta I_{\mathrm{ax}}(t)$ and $I_{\text {lat }}(t)$ are named axial and lateral currents since they stand for the sum and the difference of the left and right currents flowing across the same macro-electrode. The 4 currents $\delta I_{\mathrm{ax}, 1}, \delta I_{\mathrm{ax}, 2}$, $I_{\text {lat }, 1}, I_{\text {lat }, 2}$ are gathered into a measurement vector named $M(t)$. As explained in [18], these 2 linear combinations of the raw currents allow inferring whether the object is insulating or conductive (its electric property) and if it is on the left or the right side of the sensor. In our experiments (see Fig. 1.a), the sensor is moved along a $60 \mathrm{~cm}$ length straight line in the equatorial plane of the object. The object is initially placed at the middle of this line (with a given lateral distance $d$ from it, and at the center of the 2 meters wide tank), and sufficiently far from the probe to be out of its range. The sensor is controlled by its forward velocity $V=1 \mathrm{~cm} / \mathrm{s}$ and its angular velocity $\Omega=0 \mathrm{rad} / \mathrm{s}$. They are measured online thanks to the robot gantry. The object has a conductivity $\gamma_{o}$ different from the water conductivity $\gamma$ that is assumed to be known. Due to the small range of the object electric response, we assume that if there are several objects in the scene they are sufficiently far from each other so that the robot cannot detect both of them at the same time. Due to symmetry, the estimation of the object geometry is reduced to that of its elliptic cross-section in the sensor plane. However, it is worth noting that in this article, the model used by the approach is that of a three-dimensional ellipsoidal object. Note also that our heuristic would work in the same way with curved trajectories, as soon as the probe motion is known, and the object remains in its detection range. Experiments are performed with ellipsoidal objects $(\mathrm{a}=32 \mathrm{~mm}$ and $b=16 \mathrm{~mm})$ including spherical ones $(\mathrm{r}=20 \mathrm{~mm})$. Half of them are conductive (aluminum), and the others are insulators (plastic). For each object, we perform experiments at several distances and orientations with respect to the sensor straight trajectory (see Fig. 1.b and Fig. 1.c).

\section{B. Model of electric measurements}

Let us consider an object in a conductive medium bounded by several electrodes that are set under voltage. Integrating the governing electro-static equations of this problem for arbitrary geometries, i.e. solving the direct (electro-static) problem, 

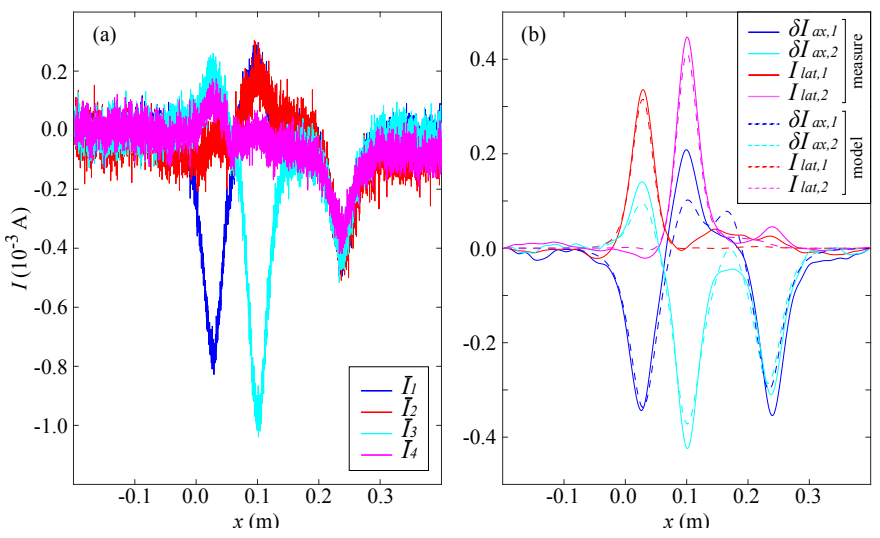

Fig. 2. Illustration of the 4 electrical currents measured along a trajectory for an insulating sphere at a lateral distance $d=40 \mathrm{~mm}$ in salt water. (a) 4 raw currents measured. (b) 4 filtered and combined currents with their associated model estimations. In this case, the model score is equal to 0.267 .

needs resorting to numerical methods as the Boundary Element Method (BEM). However, when the sensor is slender, one can address this direct problem analytically as it has been done in [18] for spherical objects. Hereafter, we use the analytical model of our sensor but with ellipsoidal objects. It is worth noting that the choice of ellipsoidal objects is of particular relevance for E-sense since in a uniform field, any object responds at leading order, as an equivalent ellipsoid [15], [19]. Mathematically, this electric response is modeled by the firstorder generalized polarization tensor of the object [20]. This model allows defining the function $f$ that gives the electric currents vector $\widehat{M}(t)$ for an object $O$ and a robot pose $S(t)$. For each $t \in 1, \ldots, n$, we thus have:

$$
\begin{gathered}
\widehat{M}(t)=f(S(t), O)=\left(\begin{array}{c}
\delta I_{\mathrm{ax}, 1}(S(t), O) \\
\delta I_{\mathrm{ax}, 2}(S(t), O) \\
I_{\mathrm{lat}, 1}(S(t), O) \\
I_{\mathrm{lat}, 2}(S(t), O)
\end{array}\right), \text { with: } \\
\delta I_{\mathrm{ax}}(S(t), O)=-\frac{1}{4 \pi \gamma} C_{0} G R_{\theta_{s}} P R_{\theta_{s}}^{t} G^{t} C_{0} U \\
I_{\text {lat }}(S(t), O)=-\frac{1}{4 \pi} P_{\perp} H R_{\theta_{s}} P R_{\theta_{s}}^{t} G^{t} C_{0} U
\end{gathered}
$$

where $\gamma$ is the conductivity of the medium, $\delta I_{\mathrm{ax}}$ and $I_{\text {lat }}$ are our current vectors, $U$ is the voltage vector, $C_{0}$ and $P_{\perp}$ are two matrices that define the robot morphology, $P$ is the polarization tensor that encodes the object electric response in its proper frame, $R_{\theta_{s}}$ is a rotation matrix where the angle between the robot and the major axis of ellipsoid is noted $\theta_{s}=\theta-\theta_{r}$. Finally, $G$ and $H$ are two matrices that depend on the distances between the object center and the electrodes. The vector $C_{0} U$ is named axial basal (or "background") currents. More details are given in Appendix A.

\section{Score for assessing the fit of measurement models}

Problem statement: Considering $t$ vectors of electric currents $M(t)$, and their associated robot poses $S(t)$, we seek for the location, size and orientation of the object equivalent ellipsoid that responds to the E-field generated by the sensor, i.e. we seek for:
- the $2 \mathrm{D}$ position $x, y$ and the orientation $\theta$ of the elliptic cross-section,

- the size of the elliptic cross-section with $a, b$ its semimajor and semi-minor axis,

- the electric conductivity $\gamma_{o}$ of the object, assumed to be conducting or insulating.

To assess the discrepancies between model-based estimation of the measurements and their real values along a trajectory, we use the function $g$ (inspired by a fitting criterion proposed in [21]):

$$
g(\mathbf{M}, \widehat{\mathbf{M}})=\frac{1}{n} \frac{\sum_{t=1}^{n}\left(\sum_{i=1}^{4}\left|M_{i}(t)-\widehat{M}_{i}(t)\right|\right)}{\sum_{t=1}^{n}\left(\sum_{i=1}^{4}\left|M_{i}(t)\right|\right)}
$$

with $\mathbf{M}=(M(1), M(2), \ldots M(t))$ the vector of measurements, and $\widehat{\mathbf{M}}=(\widehat{M}(1), \widehat{M}(2), \ldots \widehat{M}(t))$, the vector of estimations. The function $g$ is a fitting score defined as the mean of the errors between the real measures and their modelbased prediction over a set of time samples. For each time index $t$, the errors for each $\delta I_{\mathrm{ax}}(t)$ and $I_{\text {lat }}(t)$ are summed and normalized to ensure the error on $\delta I_{\mathrm{ax}}(t)$ and $I_{\text {lat }}(t)$ to have the same contribution to the score. A score of 0 and $\geq 1$ stands for a perfect and a poor fitting respectively. For the purpose of illustration, in Fig. 2 we represented the measured and modeled currents for an insulating sphere. In this example, the estimation fits well the electric measurements while the score is about 0.267 . This score is not equal to zero because of the model approximations and experimental errors. The main modeling error is due to the fact that the model neglects the object response beyond its dipolar components in spite of its non-negligible size with respect to the basal field, especially when it is close to the robot. Other errors originate from the experimental set up itself: (i) the rod supporting the object has its own electrical response, (ii) the object pose cannot be perfectly known (it is estimated in situ before each experiment by remote controlling the robot up to touch the object on each side), (iii) the conductivity in the tank is not uniform (but depends on temperature, salinity) and is measured by a conductometer (WTW197i) with $0.5 \%$ accuracy. It is difficult to separate and quantify these errors as they are mixed together. To assess the influence of each parameter on $g$, the model parameters are varied in (4) while the real measurements are maintained fixed. The results of this study are presented in Appendix B. In short, we found that the angle of the ellipsoid weakly influences the score. However, an error on the $x$-axis (longitudinal) has a strong influence as it produces a phase shift of the signal. Finally, an error on the $y$ axis or on the object size has the most critical impact on $g$ as it drastically affects the object response by changing the distance object/robot or the object's volume. These tendencies will be confirmed by the experimental results (see Sec. V), where we will see that the parameter that has the smallest influence on $g$, i.e. the angle of the ellipsoid, is the most difficult to estimate.

\section{A MODEL-BASED HEURISTIC}

\section{A. Overview of the algorithm}

As the problem is strongly nonlinear, solving the inverse problem is difficult and may be impossible. The idea behind the 


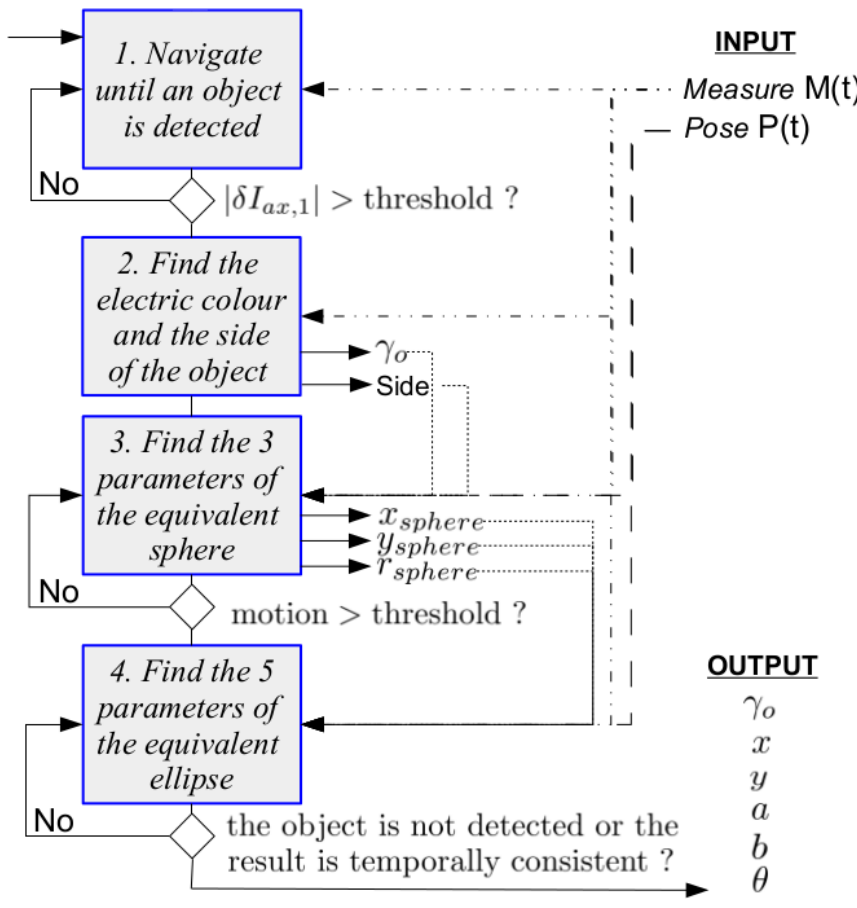

Fig. 3. Overview of the heuristic estimating the object pose and size.

development of an exhaustive grid search heuristic comes from the following facts. First, the direct problem can be solved fast by using the analytical model. Second, all parameters are bounded in small intervals which reduce the computational efforts of a systematic exploration of the space of candidates. The location ( $x, y$ coordinates) is constrained by the limited range of the electric sensor due to the rapid decrease of the E-field (the maximum detection range is below one robot length). Regarding the size parameters $(a, b)$, they need to be consistent with the assumptions of the dipolar model [18], i.e., they should be below $1 \mathrm{~cm}$. However, our experiments have shown that the model remains reliable for an object whose size is below $1 / 4$ of the robot length $(5 \mathrm{~cm})$. Finally, in our experiments, we consider that the orientation $\theta$ of the object ranges in $[0, \pi]$ and the object conductivity $\gamma_{o}$ is either equal to $1 e^{5}$ for conductive objects, or $1 e^{-5}$ for insulating ones [13]. The parameter space being bounded, when a perturbation on the electric measurements is detected, our method consists in discretizing the object parameters interval, to create a base of candidate solutions, and to test each of them to find the best fitting between estimations and measurements (the lower $g$ ). All the scores for all candidate solutions are updated each time a new measurement is recorded. This heuristic can be decomposed in 4 main steps presented in Fig. 3:

1) Navigate until a perturbing object can be detected,

2) Retrieve the robot side on which the object is and identify whether it is insulating or conducting,

3) Create the first candidate base, and estimate the location and size of a sphere that best explains the measurements,

4) From this first approximation, create the second base of candidates and estimate the final size and pose of an equivalent ellipsoid.

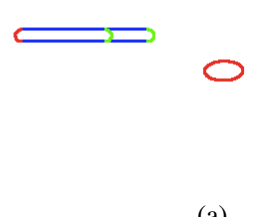

(a)

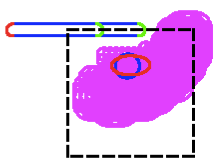

(c)

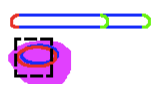

(e)

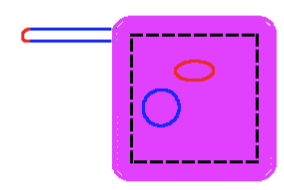

(b)

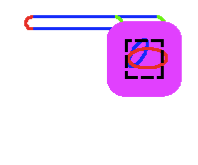

(d)

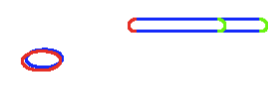

(f)
Fig. 4. Pose and size estimation of a conductive ellipsoid $(32 \times 16 \mathrm{~mm})$ with $d=50 \mathrm{~mm}$ and $\theta=0$ in salt water. Score of the model using the ground truth parameters (0.313). Real object (red), estimated object (blue), all candidate solutions (purple). a) Navigate until the object is detected ( $t=1$ to $t=t_{1}$ ), b) Get its side, its electrical properties and create the sphere base $\left(t=t_{1}\right)$, c) Estimate the sphere, d) Create the ellipsoid base $\left(t=t_{2}\right)$, e) Estimate the ellipsoid, f) Select the smallest score (0.293).

The sphere-based location stops when the robot has navigated over a fixed distance after detection (explained in Sec. IV-D). The estimation of the ellipsoid stops when it remains consistent after several sensor positions, or in the worst case when the perturbation of the object has disappeared. The solution obviously depends on both the space discretization parameters (set by the user) and the trajectory of the robot (a few centimeters). The discretization parameters and the trajectory are studied in Sec. V-E.

\section{B. Object detection}

The first stage consists of the detection of the perturbing object. When there is no perturbation of the E-field we have $\left|\delta I_{\mathrm{ax}, \mathrm{i}}\right| \approx\left|I_{\mathrm{lat}, \mathrm{i}}\right| \approx 0$. While the sensor goes forward, it discovers its environment with its 2 front electrodes (see Fig. 4.a). As soon as $\left|\delta I_{\mathrm{ax}, 1}\right|$ becomes greater than a fixed threshold, an object is detected. This threshold is set as twice the noise level and fixed once for all since the noise level does not depend on $\gamma$ [17]. At this instant, we note $t=t_{1}$.

\section{Determination of the electric properties of the object}

When an object is detected (see Fig. 4.b), depending on the sign of $\delta I_{\mathrm{ax}, 1}$ we can infer on which side (left or right) of the sensor, the object is, along with its electric properties $\gamma_{o}$. There are 4 possible scenarios detailed in [13]. Then, we assign the intervals of the location of the equivalent sphere such as:

$$
\begin{aligned}
& \text { - } x_{\text {sphere }} \in\left[x_{r}\left(t_{1}\right)+l_{F} / 4, x_{r}\left(t_{1}\right)+l_{F} / 4+l_{F}\right] \text {, } \\
& \text { - } r_{\text {sphere }} \in\left[0, l_{F} / 4\right],
\end{aligned}
$$

If $\delta I_{\mathrm{ax}, 1}<0$ (respectively $\delta I_{\mathrm{ax}, 1}>0$ ), the object is insulating (resp. conducting):

$$
\text { - } \left.\gamma_{o}=1 e^{-5} \text { (resp. } \gamma_{o}=1 e^{5}\right) \text {. }
$$

If $\delta I_{\mathrm{ax}, 1}, I_{\mathrm{lat}, 1}>0$ (resp. $\delta I_{\mathrm{ax}, 1}, I_{\mathrm{lat}, 1}<0$ ), a conductive object (resp. insulating) is on the left. 
- $y_{\text {sphere }} \in\left[y_{r}\left(t_{1}\right), y_{r}\left(t_{1}\right)+l_{F}\right]$.

If $\delta I_{\mathrm{ax}, 1}>0, I_{\mathrm{lat}, 1}<0$ (resp. $\delta I_{\mathrm{ax}, 1}<0, I_{\mathrm{lat}, 1}>0$ ), a conductive object (resp. insulating) is on the right.

- $y_{\text {sphere }} \in\left[y_{r}\left(t_{1}\right), y_{r}\left(t_{1}\right)-l_{F}\right]$.

The $x$ and $y$ intervals define a rectangle depicted in black dot in Fig. 4.b.

\section{Estimation of the equivalent sphere}

The continuous intervals of $\left(x_{\text {sphere }}, y_{\text {sphere }}, r_{\text {sphere }}\right)$ are changed into grids of nodes separated by 3 steps $\epsilon_{x}, \epsilon_{y}, \epsilon_{r}$ set by the user with $\epsilon_{x}=\epsilon_{y}$. This discretization provides a set $O=\left\{O_{i}\right\}, i \in\left\{1, \ldots, n b_{\text {sphere }}\right\}$ of triplets gathering the 2 coordinates and radius of the candidate spheres (see Fig. 4.b). Applying the $f$ function (1) to each $O_{i}$, allows estimating the vector $\overrightarrow{\mathbf{M}}$ that would be measured by the robot for such an object along the trajectory $S$. Then, the sphere parameters that best fit the electric measurements are selected by scoring each $O_{i}$ with the $g$ function (4). This heuristic selects the spherical object model that minimizes $g$ after a certain amount of different positions (see Fig. 4.c). The displacement required to switch from sphere to ellipsoid estimation was set empirically to $4 \mathrm{~cm}$ in accordance with the robot speed and data acquisition rate we used. At this step, we note $t=t_{2}$ with $t_{1} \leq t_{2} \leq n$. The lower score sphere approximation defines the triplet noted ( $\left.x_{\text {sphere }}, y_{\text {sphere }}, r_{\text {sphere }}\right)$. It is computed as follows:

$$
\underset{i \in\left\{1, \ldots, n b_{\text {sphere }}\right\}}{\operatorname{argmin}} g(\mathbf{M}, \widehat{\mathbf{M}}) \text { with a spherical model. }
$$

\section{E. Estimation of the equivalent ellipsoid}

Based on the above sphere approximation, at $t=t_{2}$ a second set $O$ of ellipsoidal candidates is generated. Applying the same method to the remaining points of the trajectory allows refining $(x, y)$, while providing the 3 other parameters $(a, b, \theta)$ (that define the object ellipsoidal estimation).

- $x \in\left[x_{\text {sphere }} \pm l_{F} / 20\right]$,

- $y \in\left[y_{\text {sphere }} \pm l_{F} / 20\right]$,

- $\theta \in[0, \pi]$

- $a, b \in\left[r_{\text {sphere }} \pm l_{F} / 16\right]$ with $a \geq b$.

In this second estimation, the resolution of the $x, y$ and sizeparameters grid is twice higher than for the sphere, i.e. one sets 4 steps $\epsilon_{x}, \epsilon_{y}, \epsilon_{a}, \epsilon_{b}$ where $\left(\epsilon_{x}, \epsilon_{y}\right)$ are half those of the sphere, and $\epsilon_{a}=\epsilon_{b}=\epsilon_{r} / 2$. The estimation of $\theta$ requires a further discretization parameter $\epsilon_{\theta}$. This discretization provides a second set $O$ gathering the vectors of ellipsoid parameters $O_{j}$, with $j \in\left\{1, \ldots, n b_{\text {ellipsoid }}\right\}$. This stage corresponds to the Fig. 4.d .e .f. Finally, the algorithm ends by minimizing $g$ again, but now using the model of the ellipsoid instead of that of the sphere in (5).

\section{F. Score illustration with experimental data}

Fig. 4 illustrates the approach applied to a $32 \times 16 \mathrm{~mm}$ conductive ellipsoid at $50 \mathrm{~mm}$ in salt water. To supplement this, in Fig. 5, we show the results of the algorithm applied to an insulating ellipsoid at $50 \mathrm{~mm}$ in salt water. The measured electric currents on the first macro-electrode used to feed the heuristic
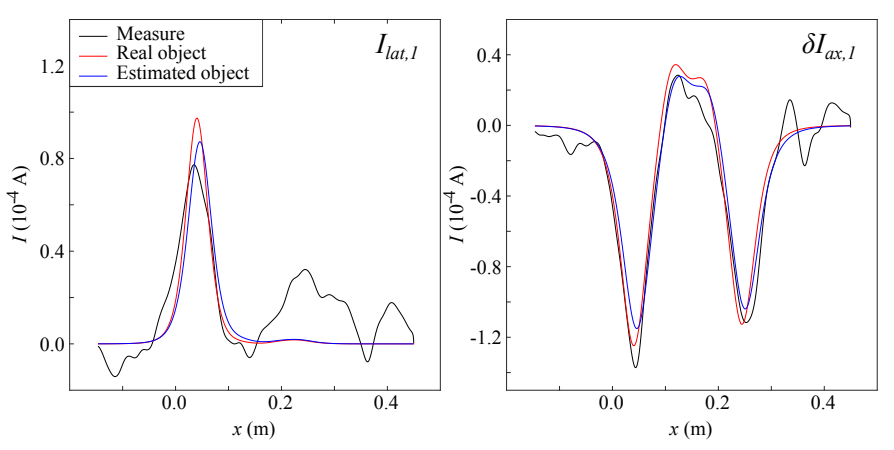

(a)

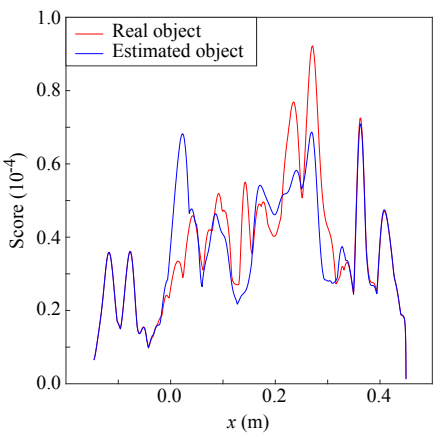

(b)

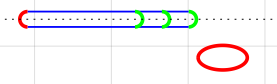

(d)

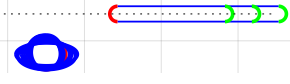

(f)

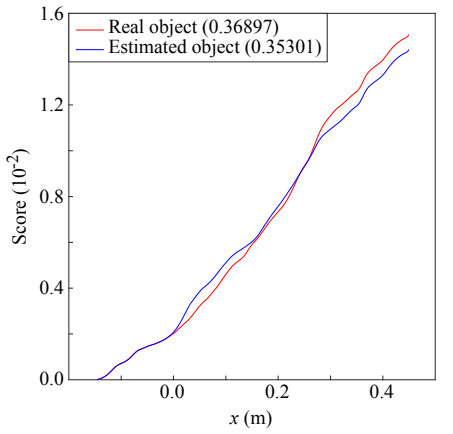

(c)

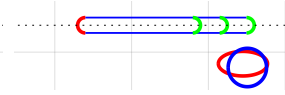

(e)

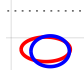

(g)

Fig. 5. Pose and size estimation for an insulating ellipsoid $32 \times 16$ with $d=50 \mathrm{~mm}$ in salt water. This ground truth ellipsoid is depicted in red and the estimated model in blue. a) Currents $I_{\mathrm{lat}, 1}$ and $\delta I_{\mathrm{ax}, 1}$ measured by the robot together with the real and estimated models , b) Step by step score for the real object model and the estimated model along the full trajectory, c) Cumulative score for the model resulting from the heuristic (blue) compared to the real object model cumulative score (red), d) Position of the probe when it detects the object and starts the location and size estimation of a spherical model $\left(t=t_{1}\right)$, e) Result of the estimation of a sphere in blue $\left(t=t_{2}\right)$, f) The 10 ellipsoids that have the best score at the end of the heuristic $g$ ) Ellipsoid with the minimum score at the end of the heuristic.

are plotted in Fig. 5.a in black. The currents corresponding to the real (ground truth) model are in red, while those estimated by our heuristic are in blue. In Fig. 5.b and.c we show the step by step evolution of the score and the cumulative score for the real model and the best estimated model along the trajectory. As it can be seen, the algorithm found a model that better fits the measurements than those calculated with the real object parameters, which is due to non-negligible modeling and experimental approximations. The fitting score (cumulative score) of the best estimated ellipsoid is (0.353) while the score calculated with the real model is (0.368). Finally, the parameters of the estimated elliptic equatorial section $(x, y, a, b, \theta)$ are $(0.149,-0.215,0.027,0.02,0)$ while the real one are $(0.145,-0.215,0.032,0.016,0)$ (see Fig. 5.g). In Fig. 5.d we present the position of the probe when it detects the object (step 1 of the heuristic). At this step, the spherical 


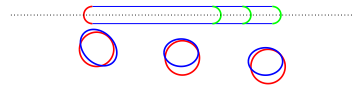

(a)

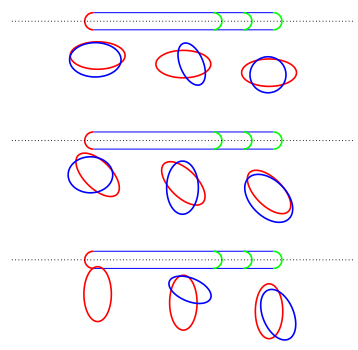

(c)

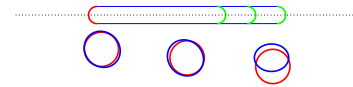

(b)

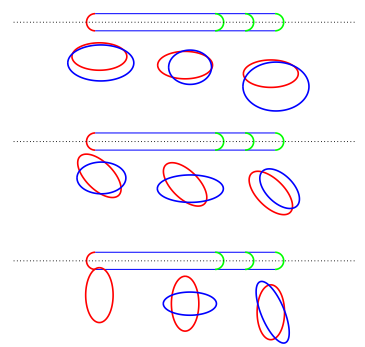

(d)
Fig. 6. Pose and size estimation of spherical $(r=20 \mathrm{~mm})$ and ellipsoidal objects ( $a=32 \mathrm{~mm}, b=16 \mathrm{~mm})$ in salt water. Real object (red) and estimated object (blue). The columns display 3 different lateral distances $d=40,50,60 \mathrm{~mm}$. (a) 3 experiments with a conductive sphere, (b) 3 experiments with an insulating sphere, (c) 9 experiments with a conducting ellipsoid, (d) 9 experiments with an insulating ellipsoid. In (b) and (c) the 3 rows display different ellipsoid orientations $\left(0^{\circ}, 45^{\circ}, 90^{\circ}\right)$.

TABLE I

POSE AND SIZE ESTIMATION RESULTS CORRESPONDING TO THE FIGURES 6 AND 7 WITH $\epsilon_{x}=\epsilon_{y}=\epsilon_{a}=\epsilon_{b}=0.0025, \epsilon_{\theta}=22.5$.

\begin{tabular}{c|c|ccc}
\hline \hline & & $E_{\ell}(\mathrm{m})$ & $E_{s}(\%)$ & $E_{\theta}\left(^{\circ}\right)$ \\
\hline & 3 expe. (insu. spheres) & 0.0044 & 7.0 & - \\
& 3 expe. (cond. spheres) & 0.0053 & 11.2 & - \\
SALT & all spheres expe. & 0.0048 & 9.1 & - \\
WATER & 8 expe. (insu. ellipsoids) & 0.0076 & 18.9 & 25.3 \\
$1.6 \mathrm{~S} / \mathrm{m}$ & 8 expe. (cond. ellipsoids) & 0.0084 & 16.6 & 30.9 \\
& all ellipsoids expe. & 0.0080 & 17.7 & 28.1 \\
& 16 expe. (insu. ellipsoids) & 0.0088 & 22.8 & 30.4 \\
FRESH & 16 expe. (cond. ellipsoids) & 0.0111 & 18.0 & 11.4 \\
WATER & all ellipsoids expe. & 0.0099 & 20.4 & 20.9 \\
$0.04 \mathrm{~S} / \mathrm{m}$ & \multicolumn{4}{l}{}
\end{tabular}

model-based estimation is started. Fig. 5.e illustrates the result of this equivalent sphere estimation (step $\mathbf{3}$ of the algorithm). Fig. 5.f shows the 10 best-scored ellipsoids provided by the algorithm. This figure illustrates the complexity of the electric inverse problem. Indeed, even after a large displacement (more than one robot length), the algorithm found an acceptable location but many different sizes and orientations remain with very close scores. This is explained by the fact that size, orientation and location errors can compensate each other.

\section{EXPERIMENTAL RESULTS}

\section{A. Overview of the experiments}

To illustrate the effectiveness of our approach, we processed 54 experiments ( 22 in salt water, 32 in fresh water). For all these experiments, we used the same discretization parameters, empirically chosen as indicated in Sec. V-F. These parameters are $\epsilon_{x}=\epsilon_{y}=\epsilon_{a}=\epsilon_{b}=0.0025 \mathrm{~m}$ and $\epsilon_{\theta}=22.5^{\circ}$, which implies an average number of tested models per experiment, about $5.510^{4}$. All the results were generated off-line on a laptop computer equipped with an Intel Dual Core i7 $2.2 \mathrm{GHz}$ CPU. For each experiment, the computational time under

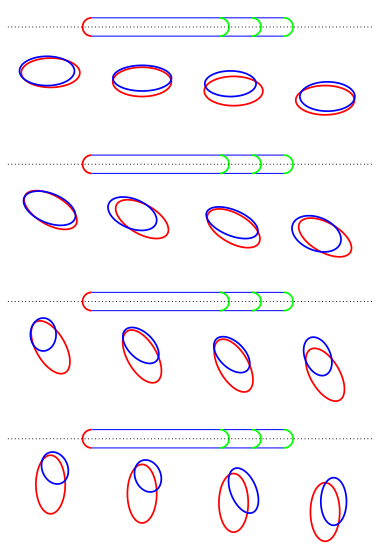

(a)

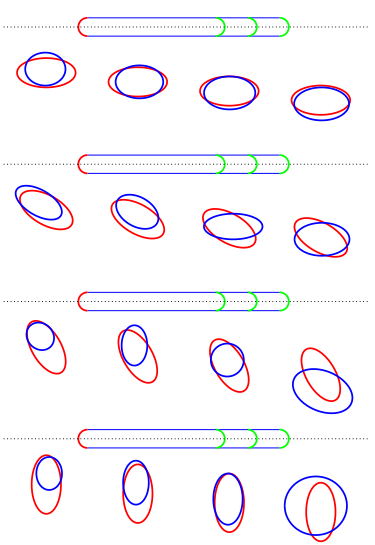

(b)
Fig. 7. Pose and size estimation of ellipsoidal objects $(a=32 \mathrm{~mm}, b=$ $16 \mathrm{~mm}$ ) in fresh water. Real object (red) and estimated object (blue). The columns display 4 different lateral distances $(50-60-70-80 \mathrm{~mm})$, and the rows, 4 ellipsoid orientations $\left(0^{\circ}, 30^{\circ}, 60^{\circ}, 90^{\circ}\right)$. a) 16 experiments with a conductive ellipsoid, b) 16 experiments with an insulating ellipsoid.

Matlab for the whole trajectory was about 80 s (in comparison the experiment duration was about $60 \mathrm{~s}$ ). To quantify the estimation results, we used the 3 following metric errors of [13] defined by:

- $E_{\ell}=\sqrt{\left(x-x_{\text {real }}\right)^{2}+\left(y-y_{\text {real }}\right)^{2}}$,

- $E_{\theta}=\left|\theta-\theta_{\text {real }}\right|$,

- $E_{s}=\sqrt{\left(\left(a-a_{\text {real }}\right)^{2}+\left(b-b_{\text {real }}\right)^{2}\right) /\left(a_{\text {real }}^{2}+b_{\text {real }}^{2}\right)}$, with $(x, y, a, b, \theta)$ and $\left(x_{\text {real }}, y_{\text {real }}, a_{\text {real }}, b_{\text {real }}, \theta_{\text {real }}\right)$ the parameters of the estimated and the real object model, while the subscripts $\ell, \theta$ and $s$ are related to the "location", the "orientation" and the "size" respectively.

\section{B. Results in salt water}

Fig. 6.a (resp .b), shows the location and size estimation errors obtained for a conductive (resp. insulating) sphere, by using the ellipsoid model. For each sphere, we conducted 3 experiments at different distances and obtained an average error on the size $\bar{E}_{s} \simeq 9 \%$, and on location $\bar{E}_{\ell} \simeq 0.0048 \mathrm{~m}$ (see Tab. I). As expected, the objects are well located and the size of the estimated ellipsoid is close to the sphere shape. Similar experiments are illustrated in Fig. 6.c and .d, but for 16 experiments performed with our 2 ellipsoidal objects (one conductive, one insulating). For each of both, we tested 3 different distances and 3 different orientations $\left(0^{\circ}, 45^{\circ}, 90^{\circ}\right)$. In Fig. 6.c and .d, one experiment is missing at $40 \mathrm{~mm}$ (last row) since the robot was colliding with the object. In this second case, we obtained $\bar{E}_{s} \simeq 17 \%, \bar{E}_{\ell} \simeq 0.008 \mathrm{~m}$ and $\bar{E}_{\theta} \simeq 28^{\circ}$.

\section{Results in fresh water and comparison with [13]}

To supplement the above results in salt water, we show in Fig. 7, 32 experimental results obtained with the same ellipsoids but in fresh water. In this case, $\bar{E}_{s} \simeq 20 \%, \bar{E}_{\ell} \simeq 0.009 \mathrm{~m}$ and $\bar{E}_{\theta} \simeq 20^{\circ}$ (see Tab. I). These results are globally less good than in salt water. However, this is only due to the fact that we performed more experiments at larger range (see Sec. V-D). To 
perform these experiments, we used the data produced in our previous paper [13]. Results are consistent with those of [13]: $\bar{E}_{s} \simeq 20 \%$ with the heuristic, against $\bar{E}_{s} \simeq 16 \%$ (and $10 \%$ after a reactive alignment in [13]); $\bar{E}_{\theta} \simeq 21^{\circ}$ in this paper against $\bar{E}_{\theta} \simeq 27^{\circ}$ without alignment in [13]. Finally, even if the estimation of the size is not as good as in [13], these results make sense since we estimate the 6 object parameters in parallel with a unique algorithm, while when estimating the size in [13], the ground truth location is supposed to be known after the location or an alignment phase.

\section{Discussion about the heuristic results}

The heuristic has similar performances in salt and fresh water except for the range, that was reduced in salt water. This difference was due to the hardware. Our electronic board being designed for fresh water, was saturated by the increase of currents in highly conductive salt water. To avoid this saturation in salt water, the emitting voltage had to be decreased which resulted in a diminution of the range. In the future, we plan to develop a new electronic board able to self-adapt to a wider range of conductivity. Regarding the results of the heuristic in itself, we observe the same characteristics as in [13] that we now briefly remind. Size and location are rather well estimated even if the algorithm tends to underestimate the distance and the size. Orientation remains difficult to estimate as this is confirmed by the weak sensitivity of our score to $\theta$ (see Appendix B). The results are better for conductive than for insulating objects and are improved when the objects get closer to the sensor, except when $\theta$ approaches $90^{\circ}$ (last rows of Fig. 6 and 7). In this pathological case, the quadripolar response of the object which is ignored by our dipolar model has a non-negligible effect on the measurements and introduces a strong bias in the estimates of the algorithm. Finally, when the object is pushed away from the probe, the influence of the noise increases and the performance of the algorithm obviously drops, this phenomenon being reinforced in salt water for the aforementioned reasons.

\section{E. Influence of the robot location errors on the results}

In the previous part, we have shown results obtained using different datasets including different objects, distances, and orientations. To get a meaningful evaluation of the repeatability of the heuristic, we repeated several times the same experiment to check if the results were consistent. We arbitrarily selected one experiment (insulating ellipsoids at $50 \mathrm{~cm}$ with an orientation angle of $0^{\circ}$ ), and we performed 15 times the same straight trajectory in our salt water tank. Due to the imprecision of the robot gantry and to the heterogeneity of the salinity in the water tank, we obtained slightly different currents measures (see the $15 I_{\text {lat,1 }}$ signals in Fig. 8.a). These different currents explain the differences between results displayed in the histograms (see Fig. 8.b). Globally, the location error varies of $\pm 3 \mathrm{~mm}$, the size estimation of $\pm 6 \%$ and the orientation error that was always critical, varies of $\pm 30^{\circ}$. These results are consistent and confirm the repeatability of the heuristic. To go further and assess whether a robot equipped with a more imprecise onboard location system, can also use such a

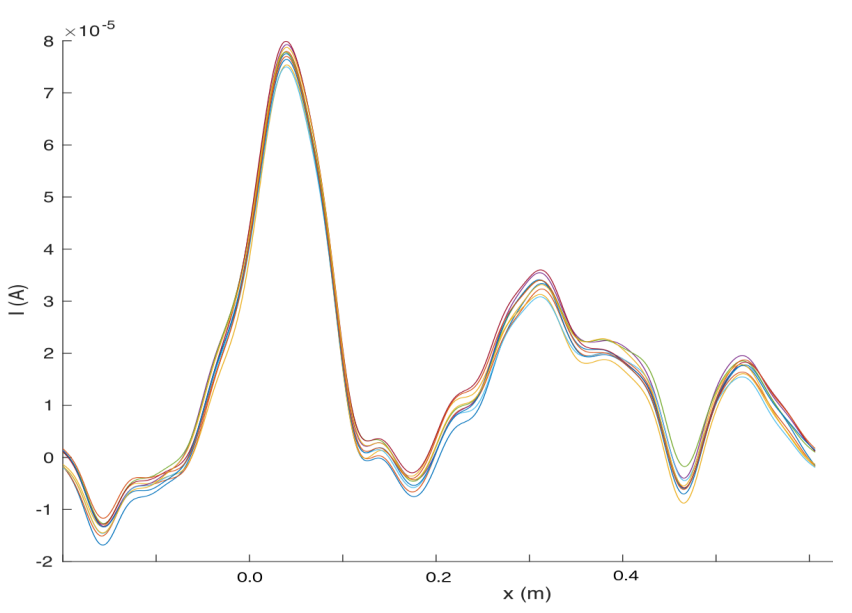

(a)

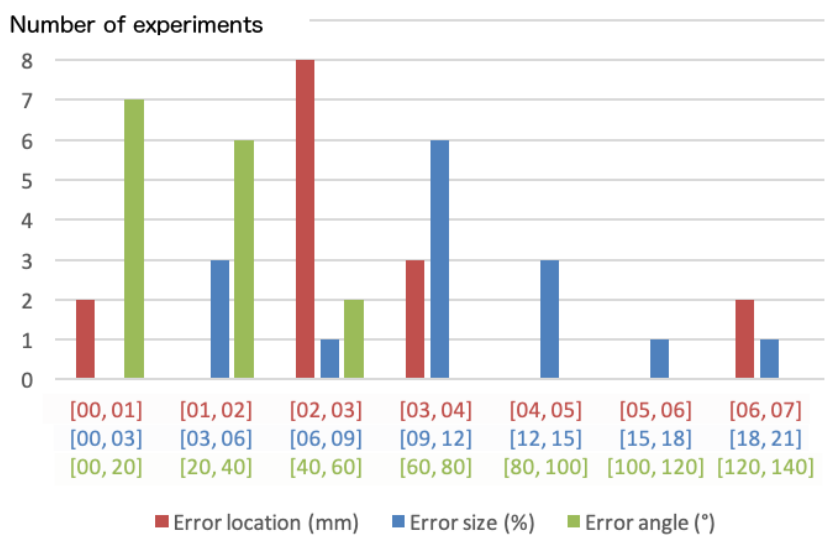

(b)

Fig. 8. Results obtained by repeating 15 times a trajectory with an insulating ellipsoid $(32 \times 16 \mathrm{~mm}), d=50 \mathrm{~cm}$ and $\theta=0$ in salt water. a) Differences on $I_{\text {lat, } 1}$ for the 15 experiments b) Histograms of location, size and angle errors for these 15 experiments.

heuristic, we added noise with a normal distribution and zero mean on the sensor velocity $V$, as well as on the heading $\Omega$. The standard deviation of the noise was the same as for the linear velocity and the heading. In Tab. II and Fig. 9 we show that as the standard deviation of the noise increases, the quality of location and size estimation results drops, which makes sense since the current measurements do not correspond to the models anymore.

\section{F. Influence of the heuristic parameters on the results}

We close the discussion by assessing the influence of the heuristic parameters on the results. In Tab. III and Fig. 10, we show that increasing the discretization, rapidly impacts the computation time but does not change much the results. The parameters presented in Table III column 3 are the best compromise between time and accuracy. This explains why we chose these values to perform all the experimental results presented in the paper. Table III, column 1, also shows that using the sphere model to approximate an ellipse gives an important error on the size estimation, but a precise location by testing significantly fewer models. This point confirms the validity of the strategy which consists first to roughly estimate 
TABLE II

EXPERIMENT WITH AN INSULATING ELLIPSOID AT 50 MM WITH NOISE ON THE MEASURE OF THE MOTION.

\begin{tabular}{c|cccc}
\hline \hline Noise on $V$ and $\Omega$ & 0 & 10 & 20 & 30 \\
\hline$E_{\ell}(\mathrm{m})$ & 0.0025 & 0.0055 & 0.012 & 0.035 \\
$E_{s}(\%)$ & 16 & 12 & 9 & 33 \\
$E_{\theta}\left({ }^{\circ}\right)$ & 22.5 & 45 & 45 & 67.5 \\
Corresponding Fig. & $6 . \mathrm{d}$ & $9 . \mathrm{c}$ & $9 . \mathrm{d}$ & $9 . \mathrm{e}$ \\
\hline \hline
\end{tabular}

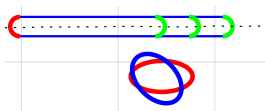

(a)

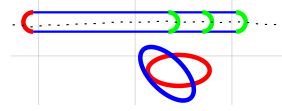

(b)

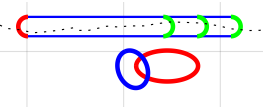

(c)
Fig. 9. Experiments with an insulating ellipsoid at $d=50 \mathrm{~mm}$ in salt water adding white noise on the robot linear and angular speed. The simulated noisy trajectory is depicted in black on each plot.

an equivalent sphere, and then to reduce the search area around this sphere location and to refine the discretization parameters.

\section{CONCLUSION}

We proposed a new model-based heuristic for object location and size approximation with electric sense. The approach is a purely model-based approach using a new dedicated score function to quantify the error between experimental and model-based measurements. This score is used as a comparison criterion to select among a pool of possible models, the model that best fits with experimental measurements. Such a score can be useful for further research in the field, e.g. to measure the quality of experimental data or to compare different models for the same dataset. As the existing methods, the three-dimensional objects are modeled by their dipolar response and excited by an active sensor sharing the same equatorial plane. While consistent with other methods, our approach contrasts with them since: (i) it works with a simple unique trajectory (alignment phases are not necessary), (ii) it does not separate location and size estimation but rather estimates all parameters in parallel, (iii) it does not require multiple polarizations of the sensor. Finally, this paper also contributes to the future application of electric sense in sea water. In the future, we plan to improve the results by

TABLE III

EXPERIMENT WITH DIFFERENT ALGORITHM PARAMETERS. THE TIME TO PERFORM THE STRAIGHT TRAJECTORY WAS $60 \mathrm{~s}$.

\begin{tabular}{c|cccc}
\hline \hline Estimated model & Sphere & Ellipsoid & Ellipsoid & Ellipsoid \\
\hline$\epsilon_{x}, \epsilon_{y}, \epsilon_{a}, \epsilon_{b}(m)$ & 0.005 & 0.005 & 0.0025 & 0.00125 \\
\hline$E_{\ell}(\mathrm{m})$ & 0.005 & 0.007 & 0.0025 & 0.0076 \\
$E_{s}(\%)$ & 35 & 13 & 16 & 4 \\
$E_{\theta}\left({ }^{\circ}\right)$ & - & 45 & 22.5 & 45 \\
Time $(\mathrm{s})$ & 57 & 26 & 90 & 397 \\
Sphere tested & 14792 & 1936 & 14792 & 108375 \\
Ellipsoid tested & - & 2574 & 10075 & 19035 \\
Corresponding Fig. & $10 . \mathrm{a}$ & $10 . \mathrm{b}$ & $10 . \mathrm{c}$ & $10 . \mathrm{d}$ \\
\hline \hline
\end{tabular}

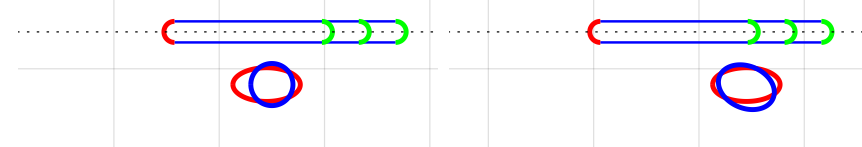

(a)

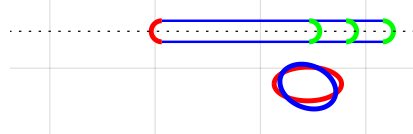

(c) (b)

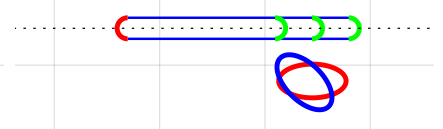

(d)
Fig. 10. Location and size estimation of an insulating ellipsoid in salt water with different algorithm parameters. The depicted robot position corresponds to the robot location when the heuristic has selected the result.

integrating the quadripolar response to our analytical model and to reduce the processing time by using intervals algebra.

\section{APPENDIX}

\section{A. Model parameters}

In this first appendix, we give the details of the matrices in (2) and (3). $C_{0}$ and $P_{\perp}$ are $4 \times 4$ matrices depending on the robot morphology, $P$ is the $3 \times 3$ matrix tensor that encodes the object electric response, $R_{\theta_{s}}$ is a rotation matrix, with $\theta_{s}=\theta-\theta_{r}$ denoting the angle between the robot and the major axis of the ellipsoid. $G$ and $H$ are $4 \times 3$ matrices modeling the influence of the distances between the object center and the electrodes. In the experimental conditions of the article, $P_{\perp}, C_{0}, G, H$ are defined as:

$$
\begin{gathered}
P_{\perp}=10^{4} \cdot \operatorname{diag}(5.29,6.74,6.95,5.52) \\
C_{0}=\frac{\gamma}{10^{2}} \cdot\left(\begin{array}{cccc}
7.65 & -3.15 & -2.31 & -2.18 \\
-3.12 & 8.39 & -3.19 & -2.07 \\
-2.29 & -3.20 & 7.80 & -2.30 \\
-2.18 & -2.09 & -2.33 & 6.60
\end{array}\right) \\
H=\left(\begin{array}{cccc}
\left(x_{o}-x_{1}\right) / r_{1}^{3} & y_{o} / r_{1}^{3} & 0 \\
\vdots & \vdots & \vdots \\
\left(x_{o}-x_{4}\right) / r_{4}^{3} & y_{o} / r_{4}^{3} & 0
\end{array}\right) \\
\left(\begin{array}{cccc}
\left(3 y\left(x_{o}-x_{1}\right)\right) / r_{1}^{5} & \left(2 y_{o}^{2}-\left(x_{o}-x_{1}\right)^{2}\right) / r_{1}^{5} & 0 \\
\vdots & \vdots & \vdots \\
\left(3 y\left(x_{o}-x_{4}\right)\right) / r_{4}^{5} & \left(2 y_{o}^{2}-\left(x_{o}-x_{4}\right)^{2}\right) / r_{4}^{5} & 0
\end{array}\right)
\end{gathered}
$$

where $k=1,2,3,4, r_{k}=\sqrt{\left(x_{o}-x_{k}\right)^{2}+\left(y_{o}\right)^{2}}$ with $x_{k}$ the axial coordinates of the electrode $\epsilon_{k}$ and $\left(x_{o}, y_{o}\right)$ the object center position in the robot frame. Note that throughout the article, we only exploited the two first receiving macroelectrodes of our 4 macro-electrode sensor. Finally, $P$ is the leading order tensor of an ellipsoidal object [19], [22]. It defines the prolate $(b=c)$ ellipsoid electrical response such as:

$$
P=V \gamma_{d} \operatorname{diag}\left(\frac{1}{\gamma+\gamma_{d} A(\eta)}, \frac{1}{\gamma+\gamma_{d} B(\eta)}, \frac{1}{\gamma+\gamma_{d} B(\eta)}\right)
$$

with $\gamma_{d}=\gamma_{0}-\gamma$ the difference between the object and water conductivities, $V=4 \pi / 3 a b^{2}$ the object volume, $\eta=a / b$ the 
TABLE IV

SPHERICAL MODEL EVALUATION

\begin{tabular}{l|ccl}
\hline \hline Sphere models & Scores & Diff. scores & Figures \\
\hline Model real object $x, y, \theta$ & 0.429 & - & Blue on all figures \\
$\mathrm{x}+1 \mathrm{~cm}$ (right) & 0.540 & 0.111 & Red on Fig. 11.a \\
$\mathrm{x}-1 \mathrm{~cm}$ (left) & 0.493 & 0.064 & Green on Fig. 11.a \\
$\mathrm{y}+0.5 \mathrm{~cm}$ (further) & 0.467 & 0.038 & Red on Fig. 11.b \\
$\mathrm{y}-0.5 \mathrm{~cm}$ (closer) & 0.682 & 0.253 & Green on Fig. 11.b \\
$\mathrm{r}+0.5 \mathrm{~cm}$ (bigger) & 1.130 & 0.701 & Red on Fig. 11.c \\
$\mathrm{r}-0.5 \mathrm{~cm}$ (smaller) & 0.648 & 0.219 & Green on Fig. 11.c \\
\hline \hline
\end{tabular}

aspect ratio of the ellipsoid. The functions $A, B$ are elliptic integrals defined by $B(\eta)=(1 / 2)(1-A(\eta))$, and:

$$
A(\eta)=\eta^{-2} \int_{1}^{+\infty} \frac{1}{t^{2}\left(t^{2}-1+\eta^{-2}\right)} d t .
$$

In the particular case of the sphere, these integrals are not needed since for $a=b, A(\eta)=B(\eta)=1 / 3$, while $P$ is simply given by:

$$
P=\gamma_{d} V \frac{3}{2 \gamma+\gamma_{0}} \operatorname{diag}(1,1,1)
$$

Moreover, in this case, owing to object's isotropy, we have $R=R^{t}=1_{3 \times 3}$.

\section{B. Influence of each parameter on the score}

In this second appendix, we study the influence of each parameter of the spherical and ellipsoidal analytical models on the fitting score. In Fig. 11.a, .b, .c, we compare the measurement of $I_{\mathrm{ax}, 1}$ with its value based on the spherical model and 3 different values of each parameter. The first value is the ground truth value, the two others are the ground-truth value to which we add or subtract a constant error. In Fig. 11.a, we modified the $x$-axis position of about $1 \mathrm{~cm}$. In Fig. 11.b, we modified the $y$-axis position of about $0.5 \mathrm{~cm}$. In Fig. 11.c we modified the sphere radius of about $0.5 \mathrm{~cm}$. Finally, in Fig. 11.d, we modified the orientation of the ellipsoid. In this last case, we used the ellipsoidal model. The associated scores corresponding to these tests are gathered in Tab. IV. As illustrated by this set of experiments, the angle of the ellipsoid has a small influence on the score. However, an error on the $x$-axis has a strong influence as it produces a phase shift on the signal. Finally, the errors on the y-axis, or on the object size, have the most critical impact on the score as they modify the object response by changing the distance object/robot or the object volume. This study confirms the difficulty to estimate the angle of the ellipsoid as this parameter has a very small influence on the score compared to the others.

\section{REFERENCES}

[1] H. W. Lissmann and K. E. Machin, "The mechanism of object location in Gymnarchus Niloticus and similar fish," Journal of Experimental Biology, vol. 35, no. 2, pp. 451-486, 1958.

[2] T. H. Bullock and W. Heiligenberg, Electroreception. Wiley Ed., 1986.

[3] G. von der Emde, "Non-visual environment imaging and object detection through active electrolocation in weakly electric fish," Journal of Computational Physiology A, vol. 192, pp. 601 - 612, 2006.
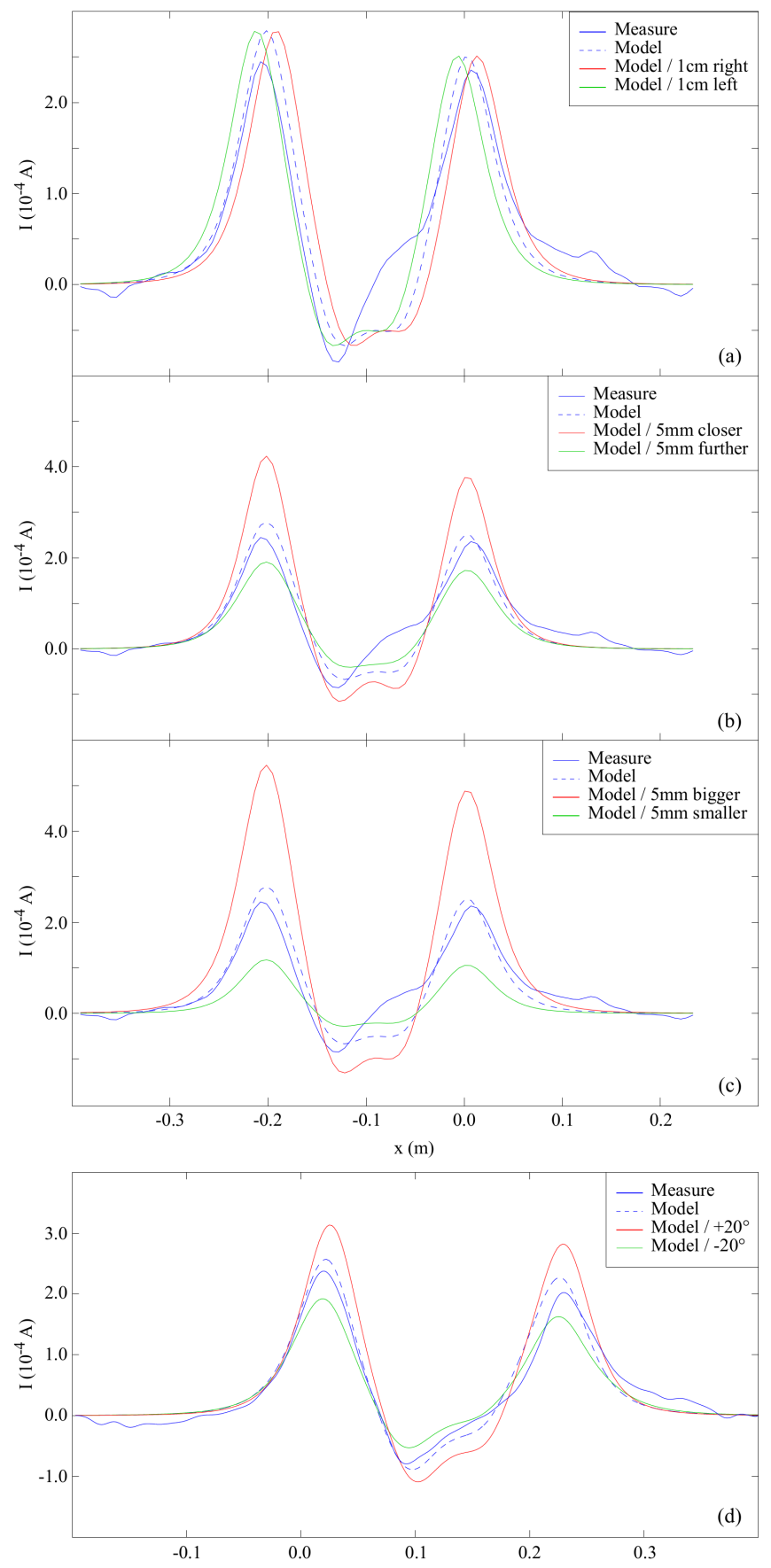

Fig. 11. Influence of the parameters on $\delta I_{\mathrm{ax}, 1}$ on the spherical and ellipsoidal models. (a) Sphere with an error on $x$-axis, (b) Sphere with an error on y-axis, (c) Sphere with an error on the sphere radius, (d) Ellipsoid with an error on the ellipsoid orientation.

TABLE V

ELLIPSOIDAL MODEL EVALUATION

\begin{tabular}{l|lcl}
\hline \hline Ellipsoids models & Scores & Diff. scores & Figures \\
\hline Model real object $x, y$, & & & \\
$a, b, \theta$ & 0.3681 & - & Blue on Fig. 11.e \\
$\theta-20^{\circ}$ & 0.422 & 0.054 & Red on Fig. 11.e \\
$\theta+20^{\circ}$ & 0.405 & 0.037 & Green on Fig. 11.e \\
\hline \hline
\end{tabular}


[4] V. Hofmann, J. I. Sanguinetti-Scheck, L. Gomez-Sena, and J. Engelmann, "From static electric images to electric flow: towards dynamic perceptual cues in active electroreception," Journal of Physiology-Paris, vol. 107, no. 1:2, pp. 95 - 106, 2013.

[5] B. Rasnow, "The effects of simple objects on the electric field of apteronotus," J. of Comparative Physiology A, vol. 3, no. 178, pp. 397411, 1996.

[6] D. Lane, "Persistent autonomy artificial intelligence or biomimesis ?" in Autonomous Underwater Vehicles (AUV), 2012 IEEE/OES, Sept 2012, pp. 1-8.

[7] F. Boyer, P. B. Gossiaux, B. Jawad, V. Lebastard, and M. Porez, "Model for a sensor inspired by electric fish," IEEE Transactions on Robotics, vol. 28, no. 2, pp. 492-505, 2012.

[8] J. R. Solberg, K. M. Lynch, and M. A. MacIver, "Active electrolocation for underwater target localization," The International Journal of Robotics Research, vol. 27, no. 5, pp. 529-548, 2008.

[9] V. Lebastard, C. Chevallereau, A. Girin, N. Servagent, P. B. Gossiaux, and F. Boyer, "Environment reconstruction and navigation with electric sense based on a kalman filter," The International Journal of Robotics Research, vol. 32, no. 2, pp. 172-188, 2013.

[10] F. Boyer, V. Lebastard, C. Chevallereau, S. Mintchev, and C. Stefanini, "Underwater navigation based on passive electric sense: New perspectives for underwater docking," The International Journal of Robotics Research, pp. 1228-1250, 2015.

[11] Y. Bai, J. Snyder, M. Peshkin, and M. A. MacIver, "Finding and identifying simple objects underwater with active electrosense," The International Journal of Robotics Research, 2015.

[12] Y. Bai, J. Snyder, Y. Silverman, M. Peshkin, and M. A. MacIver, "Sensing capacitance of underwater objects in bio-inspired electrosense," in Int. Conf. on Intelligent Robots and Systems, 2012, pp. 1467-1472.

[13] S. Lanneau, F. Boyer, V. Lebastard, and S. Bazeille, "Estimating ellipsoidal object using artificial electric sense," International Journal of Robotics Research (IJRR), 2017.

[14] H. Ammari, T. Boulier, J. Garnier, and H. Wang, "Shape recognition and classification in electro-sensing," Proceedings of the National Academy of Science (PNAS), vol. 111, no. 32, pp. 431-444, 2014.

[15] H. Ammari and H. Kang, Polarization and moment tensors, with applications to inverse problems and effective medium theory. SpringerVerlag New York, 2007.

[16] S. Bazeille, V. Lebastard, S. Lanneau, and F. Boyer, "Model based object localization and shape estimation using electric sense on underwater robots," in World Congress of the International Federation of Automatic Control, 2017.

[17] N. Servagent, B. Jawad, S. Bouvier, F. Boyer, A. Girin, F. Gomez, V. Lebastard, and P. B. Gossiaux, "Electrolocation sensors in conducting water bio-inspired by electric fish," IEEE Sensor Journal, vol. 13, no. 5, pp. 1865-1882, 2013.

[18] F. Boyer, P. B. Gossiaux, B. Jawad, V. Lebastard, and M. Porez, "Model for a sensor bio-inspired from electric fish," IEEE trans. on robotics, vol. 28, no. 2, pp. 492-505, April 2012.

[19] T. K. A. Khairuddin and W. R. B. Lionheart, "Characterization of objects by electrosensing fish based on the first order polarization tensor," Bioinspiration and Biomimetics, vol. 11, no. 5, p. 055004, 2016.

[20] H. Ammari, T. Boulier, and J. Garnier, "Modeling active electrolocation in weakly electric fish," SIAM Journal on Imaging Sciences, vol. 6, no. 1, pp. 285-321, 2013.

[21] L. Ljung, System identification (2nd ed.): theory for the user. Prentice Hall PTR, 1999.

[22] H. Ammari, J. Garnier, H. Kang, M. Lim, and S. Yu, "Generalized polarization tensors for shape description," Numerische Mathematik, vol. 126, no. 2, pp. 199-224, 2014 